\title{
Physiological evidence for diversification of IFNa- and IFN $\beta$-mediated response programs in different autoimmune diseases
}

Tamarah D. de Jong ${ }^{1 \dagger}$, Saskia Vosslamber ${ }^{1 \dagger}$, Elise Mantel', Sander de Ridder ${ }^{1}$, John G. Wesseling ${ }^{1}$, Tineke C. T. M. van der Pouw Kraan ${ }^{2}$, Cyra Leurs ${ }^{3}$, Harald Hegen ${ }^{4}$, Florian Deisenhammer ${ }^{4}$, Joep Killestein ${ }^{3}$, Ingrid E. Lundberg ${ }^{5}$, Jiri Vencovsky ${ }^{6}$, Mike T. Nurmohamed ${ }^{7}$, Dirkjan van Schaardenburg ${ }^{7}$, Irene E. M. Bultink ${ }^{8}$, Alexandre E. Voskuyl ${ }^{8}$, D. Michiel Pegtel ${ }^{1}$, Conny J. van der Laken ${ }^{8}$, Johannes W. J. Bijlsma ${ }^{7,8}$ and Cornelis L. Verweij ${ }^{1,8^{*}}$

\begin{abstract}
Background: Activation of the type I interferon (IFN) response program is described for several autoimmune diseases, including systemic lupus erythematosus (SLE), multiple sclerosis (MS), myositis (IIM) and rheumatoid arthritis (RA). While IFNa contributes to SLE pathology, IFN $\beta$ therapy is often beneficial in MS, implying different immunoregulatory roles for these IFNs. This study was aimed to investigate potential diversification of IFNa-and IFNß-mediated response programs in autoimmune diseases.
\end{abstract}

Methods: Peripheral blood gene expression of 23 prototypical type I IFN response genes (IRGs) was determined in 54 healthy controls (HCs), 69 SLE (47 test, 22 validation), 149 IFN $\beta$-treated MS (71 test, 78 validation), 160 untreated MS, 78 IIM and 76 RA patients. Patients with a type I IFN signature were selected for analysis.

Results: We identified IFNa- and IFN $\beta$-specific response programs (GC-A and GC-B, respectively) in SLE and IFN $\beta$ treated MS patients. Concordantly, the GC-A/GC-B log-ratio was positive for all SLE patients and negative for virtually all IFNß-treated MS patients, which was confirmed in additional cohorts. Applying this information to other autoimmune diseases, IIM patients displayed positive GC-A/GC-B log-ratios, indicating predominant IFNa activity. The GC-A/GC-B log-ratio in RA was lower and approached zero in part of the patients, implying relative importance of both clusters. Remarkably, GC-A/GC-B log-ratios appeared most heterogeneous in untreated MS; half of the patients displayed GC-A dominance, whereas others showed GC-B dominance or log-ratios near zero.

Conclusions: Our findings show diversification of the type I IFN response in autoimmune diseases, suggesting different pathogenic roles of the type I IFNs.

Keywords: Type I interferons, Gene expression profiling, Autoimmune diseases, Rheumatic diseases, Multiple sclerosis

\footnotetext{
* Correspondence: c.verweij@vumc.nl

tEqual contributors

'Department of Pathology, VU University Medical Center, Amsterdam, The

Netherlands

${ }^{8}$ Department of Rheumatology, Amsterdam Rheumatology and Immunology

Center, VUmc, Amsterdam, The Netherlands

Full list of author information is available at the end of the article
}

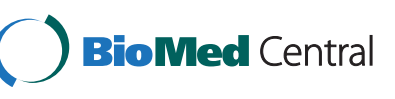

(C) 2016 de Jong et al. Open Access This article is distributed under the terms of the Creative Commons Attribution 4.0 International License (http://creativecommons.org/licenses/by/4.0/), which permits unrestricted use, distribution, and reproduction in any medium, provided you give appropriate credit to the original author(s) and the source, provide a link to the Creative Commons license, and indicate if changes were made. The Creative Commons Public Domain Dedication waiver (http://creativecommons.org/publicdomain/zero/1.0/) applies to the data made available in this article, unless otherwise stated. 


\section{Background}

Type I interferons (IFNs) comprise a large family of cytokines with antiviral, immunomodulatory and antiproliferative activities. The type I IFN family consists of 17 closely related members, including 13 IFN $\alpha$ subtypes and 4 unique members, i.e., IFN $\beta, \operatorname{IFN} \varepsilon$, IFNk and IFN $\omega$, of which IFN $\alpha$ and IFN $\beta$ are most commonly expressed and well-characterized.

Type I IFNs achieve their biological effects by binding to multi-subunit receptors, IFNAR1 and IFNAR2, on the cell surface. This leads to receptor dimerization and activation of the JAK-STAT pathway, a complex cascade of intracellular secondary messengers that emerge in transcriptional activation of genes containing IFN-stimulated response elements (ISRE) and/or IFN gamma-activated sequences (GAS) [1-4]. Upregulation of type I IFN response genes (IRGs) is referred to as a type I IFN signature and is a reflection of type I IFN bioactivity.

Initially, type I IFNs were defined by their antiviral effects and as a consequence, they were used for the treatment of chronic viral infections such as hepatitis $B$ and hepatitis $C$ [5]. The antiviral activity involves suppression of viral replication, induction of apoptosis in virally infected cells, stimulation of $\mathrm{T}$ cell and B cell responses, natural killer cell-mediated and CD8+ T cell-mediated cytotoxicity and activation of dendritic cells [6].

Increasing insight in the activities of type I IFNs has revealed their role as pleiotropic cytokines with a critical role in modulating immune responses. Several observations indicate involvement of type I IFNs and the presence of a type I IFN signature in autoimmune diseases, including systemic lupus erythematosus (SLE), Sjögren's syndrome, systemic sclerosis, multiple sclerosis (MS), idiopathic inflammatory myopathies (IIM) and rheumatoid arthritis (RA) [7-9]. Compelling evidence from studies in SLE demonstrates that IFNa in particular is directly implicated in the pathogenesis of SLE [10, 11]. SLE is characterized by the presence of autoantibodies to nucleic acid and associated proteins, which are able to induce IFN $\alpha$ protein [12]. Serum levels of IFN $\alpha$ are increased in SLE and associated with disease severity and organ involvement $[13,14]$. In support of a pathogenic role of IFN $\alpha$ in SLE was the observation that virally infected people and cancer patients treated with IFN $\alpha$ sometimes produce anti-nuclear antibodies and occasionally develop SLE-like symptoms [15, 16]. The mechanisms by which IFNa may contribute to autoimmunity are the induction of autoreactive lymphocytes, enhancement of long-term antibody responses and priming of myeloid cells.

In contrast to the pathogenic effects of prolonged IFN $\alpha$ signaling in SLE, IFN $\beta$ administration has notable therapeutic effects in MS, an autoimmune disease of the central nervous system characterized by progressive neurological dysfunction due to demyelination and axonal damage [17]. In patients with MS, treatment with IFN $\beta$ reduces clinical relapses and brain disease activity, and slows down progression of disability [18]. The antiinflammatory and tissue-protective mechanism of IFN $\beta$ likely involves anti-proliferative and pro-apoptotic effects, as well as induction of anti-inflammatory mediators such as IL-10, IL-1R antagonist and soluble TNF receptors and reduction of pro-inflammatory mediators such as IL-1, IL6 and TNF $\alpha$ [19].

From the above, the question emerges why type I IFNs can be pathogenic in SLE but therapeutic in MS. It is tempting to speculate that despite mechanistic similarities, IFN $\alpha$ and IFN $\beta$ have distinct roles in immune regulation that confer these opposing effects. Comparison of the primary amino acid sequences reveals that IFN $\alpha$ differs from IFN $\beta$ by approximately $70 \%$ [20]. Receptor binding studies demonstrate that IFN $\alpha$ and IFN $\beta$ interact with their receptors in a different manner, suggesting that IFN $\alpha$ and IFN $\beta$ activate the IFNAR1/IFNAR2-mediated signal transduction pathway in a slightly different way [21-23]. Accordingly, in vitro studies reveal that IFN $\beta$ appeared to be more potent at inhibiting cell proliferation and inducing apoptosis than IFN $\alpha$ [24]. However, it is as yet unknown whether there are differences in the downstream gene activation program of IFN $\alpha$ and IFN $\beta$-induced IFN signatures in vivo.

In the present study, we used transcript profiling to compare the IFN signature gene components regulated by IFN $\alpha$ in SLE patients to those of MS patients who were treated with IFN $\beta$. Moreover, we exploited our findings to delineate the nature of the type I IFN signature in IIM, RA and patients with MS who were IFN $\beta$ naïve.

\section{Methods}

\section{Patient recruitment}

SLE patients $(n=47)$ and RA patients $(n=76)$ were recruited at the Amsterdam Rheumatology and immunology Center, Amsterdam, The Netherlands. Patients with MS were recruited from the NABINMS study, a prospective European multicenter study that was previously described [25]. For the untreated MS cohort, blood samples collected before start of IFN $\beta$ therapy were used ( $n=160)$; for the IFN $\beta$-treated MS cohort, we used blood samples drawn after 3 months of IFN $\beta$ therapy $(n=71)$. IIM patients $(n=78)$ were recruited at the Rheumatology Unit at Karolinska University Hospital, Stockholm, Sweden or at the Institute of Rheumatology, Prague, Czech Republic, and fulfilled the diagnostic criteria for definite or probable polymyositis $(n=32)$, dermatomyositis $(n=40)$ or sporadic inclusion body myositis $(n=5)$. Healthy controls $(\mathrm{HC}, \mathrm{n}=54)$ were recruited at the VU University medical center, Amsterdam. 
This study was approved by the medical ethical committees of the VU medical center, the Slotervaartziekenhuis and Reade in Amsterdam, The Netherlands, Karolinska Hospital in Stockholm, Sweden, the Institute of Rheumatology in Prague, Czech Republic and the centers participating in the NABINMS study [25], and informed consent was obtained from all donors. Demographic data, clinical information and medication use of the patients at the time of blood sampling are shown in Table 1.

\section{Blood sampling and RNA isolation}

From the donors in the SLE, IIM, RA and HC cohorts, $2.5 \mathrm{ml}$ blood was drawn in PAXgene tubes (PreAnalytix, $\mathrm{GmbH}$, Hombrechtikon, Switzerland) and stored at $-20{ }^{\circ} \mathrm{C}$. After overnight thawing at room temperature total RNA was isolated according to the manufacturer's instructions (PAXgene Blood RNA kit). Total RNA concentration was measured using the Nanodrop spectrophotometer (Nanodrop Technologies, Wilmington, DE, USA). From the donors in the untreated MS cohort and the IFN $\beta$-treated MS cohorts, blood was collected in a Tempus tube (Applied Biosystems, Waltham, MA, USA), and processed as described before [25].

\section{Reverse transcription and pre-amplification of CDNA}

RNA $(0.5 \mu \mathrm{g})$ was reverse-transcribed into cDNA using a Revertaid H-minus cDNA synthesis kit (MBI Fermentas, Waltham, MA, USA). A single aliquot of each cDNA sample, equivalent to 12.5 ng RNA, was first subjected to 14 cycles of specific target amplification using a $0.2 \mathrm{X}$ mixture of all Taqman Gene Expression assays in combination with the Taqman PreAmp Master Mix (Applied Biosystems, Foster City, CA, USA). Following pre- amplification, the samples were diluted 1:5 (v/v) in TrisEDTA buffer, pH 8.0.

\section{Multiplex Real-Time PCR}

Custom-designed TaqMan assays for each gene were supplied by Applied Biosystems. Quantitative PCR (qPCR) analysis was performed at ServiceXS (ServiceXS B.V., Leiden, The Netherlands) using the 96.96 BioMark $^{\mathrm{mm}}$ Dynamic Array for Real-Time PCR (Fluidigm Corporation, San Francisco, CA, USA), according to the manufacturer's instructions. Thermal cycling and real-time imaging of the BioMark array was done on the BioMark instrument, and cycle threshold $\left(\mathrm{C}_{\mathrm{T}}\right)$ values were extracted using the BioMark Real-Time PCR analysis software. Relative quantities were calculated using the standard curve method, using glyceraldehyde-3phosphate dehydrogenase $(G A P D H)$ as a housekeeping gene. Expression levels were $\log _{2}$-transformed.

\section{Calculation of the type I IFN score and selection for initial analyses}

Based on whole genome expression data available in the literature, 23 interferon response genes (IRGs) (see Additional file 1: Table S1) were selected that are reflective of a communal type I IFN signature between autoimmune diseases. All 23 genes are described to be upregulated compared to healthy controls in one or more of the autoimmune diseases SLE, MS, IIM and/or RA [9, 13, 26-30]. To control for inter-experimental variation, expression levels of each gene were calculated relative to the median expression of the gene in healthy controls. Because all IRGs were strongly correlated (Pearson $r>0.7$ for $90 \%$ of the combinations, $p<0.0005)$, we calculated

Table 1 Patient characteristics for the complete cohorts or the IFN ${ }^{\text {high }}$ selection

\begin{tabular}{|c|c|c|c|c|c|c|c|}
\hline & & SLE & IFNß-treated $\mathrm{MS}^{\mathrm{b}}$ & Untreated MS & $\| \mathrm{M}$ & RA & Healthy controls \\
\hline \multirow[t]{2}{*}{ Total amount } & All & 47 & 71 & 160 & 78 & 76 & 54 \\
\hline & IFN ${ }^{\text {high }}$ & 30 & 63 & 12 & 26 & 10 & \\
\hline \multirow[t]{2}{*}{ Age in years, mean (SD) } & All & $44(14)$ & $34(8)$ & $36(10)$ & $56(14)$ & $54(13)$ & $35(10)$ \\
\hline & IFN $^{\text {high }}$ & $42(13)$ & $35(8)$ & $34(9)$ & $55(17)$ & $52(16)$ & \\
\hline \multirow[t]{2}{*}{ Female, \% } & All & 85 & 73 & 67 & 62 & 79 & 53 \\
\hline & IFN ${ }^{\text {high }}$ & 93 & 72 & 83 & 69 & 89 & \\
\hline \multirow[t]{2}{*}{ Disease activity, mean $(S D)^{a}$} & All & $4(5)$ & n.a & n.a. & n.a. & $4.8(1.4)^{c}$ & n.a. \\
\hline & IFN $^{\text {high }}$ & $5(5)$ & & & & $5.3(1.6)^{d}$ & \\
\hline \multirow[t]{2}{*}{ Current prednisolone use, \% } & All & 50 & n.a. & n.a. & 70 & 17 & n.a. \\
\hline & IFN ${ }^{\text {high }}$ & 57 & n.a. & n.a. & 60 & 22 & \\
\hline \multirow[t]{2}{*}{ Current use of other immunomodulatory drugs, \% } & All & 63 & n.a. & n.a. & 60 & 24 & n.a. \\
\hline & IFN $^{\text {high }}$ & 67 & n.a. & n.a. & 56 & 33 & \\
\hline
\end{tabular}

${ }^{2}$ Disease activity scores: for systemic lupus erythematosus (SLE), the Systemic Lupus Erythematosus Disease Activity Index; for rheumatoid arthritis, the Disease

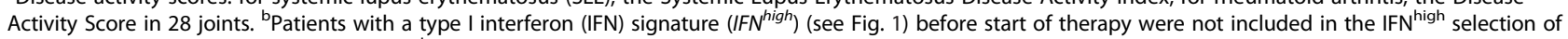

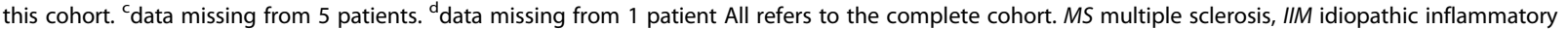
myopathies, SD standard deviation, n.a. not applicable 
an IFN score by averaging the expression levels of all IRGs per sample.

Presence of a type I IFN signature (referred to as IFNhigh) was defined as an IFN score above mean $+2 * \mathrm{SD}$ in HCs (1.3). To exclude the possibility that observed qualitative differences are actually due to quantitative differences, we selected IFN ${ }^{\text {high }}$ patients within a comparable range of IFN score, between 2.5 and 4.0, for initial analysis (Fig. 1). The remaining IFN ${ }^{\text {high }}$ patients were used as an additional cohort to verify our initial findings.

\section{Statistical analysis}

Cluster analysis was used for categorization of IRGs with respect to their relative expression between diseases [31]. TreeView was used to visualize the clustering of genes (Eisen Lab, Berkeley, CA, USA). Comparison of IRG expression between SLE and IFN $\beta$-treated MS patients was performed using the unpaired $t$ test, with multiple testing correction using the BenjaminiHochberg method. Comparison of IFN scores between SLE and MS-IFN $\beta$ was performed using the unpaired $t$ test and comparison of cluster-specific IFN scores within patients was performed using the paired $t$ test. $P$ values $<0.05$ were considered significant.

\section{Results}

\section{Differential expression of IRGs in SLE versus IFN $\beta$-treated}

\section{MS patients}

In order to explore in vivo differences in the composition of type I IFN signatures in autoimmune diseases, we studied IRG expression profiles of a prototype IFNadriven disease, i.e., SLE, and those of MS patients who were treated with IFN $\beta$ for 3 months. As described above, only patients with an IFN signature (referred to as IFN ${ }^{\text {high }}$ ) were included for further analysis. To ensure that the observed IFN signature was specifically induced by the IFN $\beta$ treatment, MS patients with an IFN signature before start of IFN $\beta$ treatment were excluded from analysis. For initial analysis, we used data from patients with comparable levels of IFN score, between 2.5 and 4.0 , as described above.

To compare the IFN signature gene components regulated by IFN $\alpha$ in SLE to those of IFN $\beta$-treated MS patients, unsupervised cluster analysis was performed (Fig. 2a). Strikingly, the analysis revealed perfect separation of SLE patients and IFN $\beta$-treated MS patients based on two IRG clusters. From the upper cluster, 5 out of 7 IRGs (GC-A) were significantly upregulated in SLE patients compared to MS-IFN $\beta$, whereas 13 out of 16 genes (GC-B) from the lower cluster were significantly upregulated in the IFN $\beta$-treated MS patients compared to SLE (Fig. 2a and Additional file 1: Table S2). GC-A and a GC-B scores were calculated by averaging expression values of these 5 and 13 genes, respectively. As shown in Fig. $2 \mathrm{~b}$, the GC-A score was significantly higher than GC-B in SLE $(p<0.001)$ whereas the GC-B score was significantly higher than GC-A in IFN $\beta$-treated MS ( $p$ $<0.001)$. Analysis of IFN ${ }^{\text {high }}$ patients with IFN scores lower than 2.5 or higher than 4.0 confirmed these findings (additional cohort, Fig. 2c). To gain insight into the relative importance of each gene cluster per patient, the GC-A/GC-B ratio was calculated. As this ratio is based on $\log _{2}$-values, a ratio above zero means a higher GC-A score compared to GC-B, whereas a ratio below zero means that the GC-B

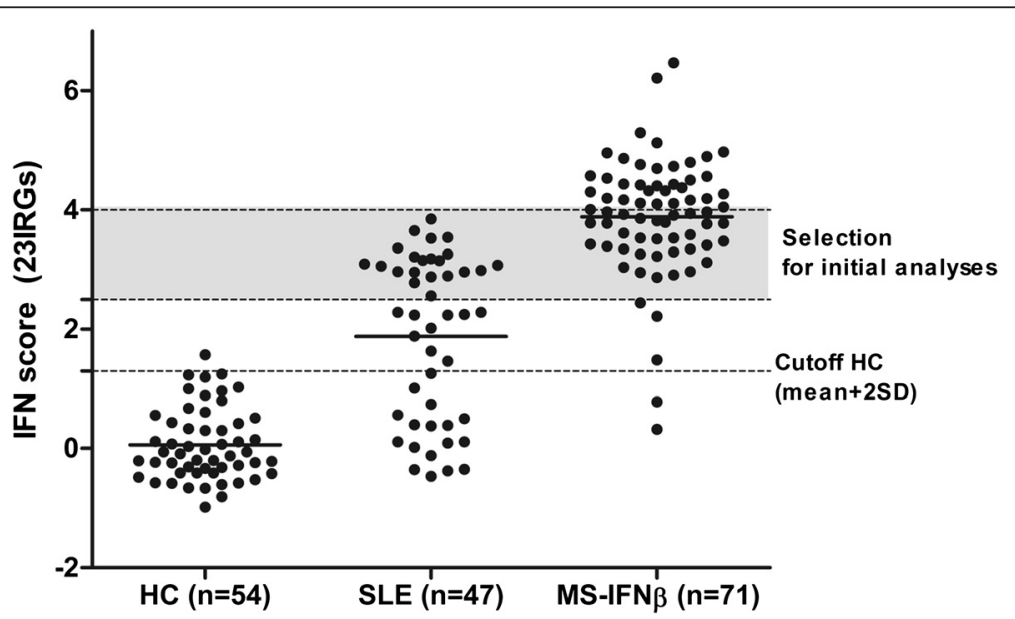

Fig. 1 Interferon (IFN) score in systemic lupus erythematosus (SLE) patients and multiple sclerosis (MS) patients who received 3 months of IFN $\beta$ treatment. Average expression levels of 23 interferon response genes (IRGS) show a comparable range for the majority of SLE

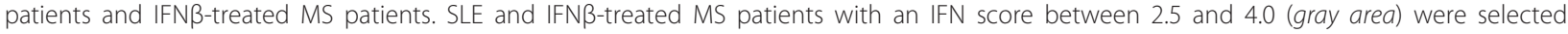
for the initial comparison of the composition of the IFN signature. Patients with a type I interferon signature and an IFN score above 4.0 or below 2.5 were used as an additional cohort. HC healthy controls 


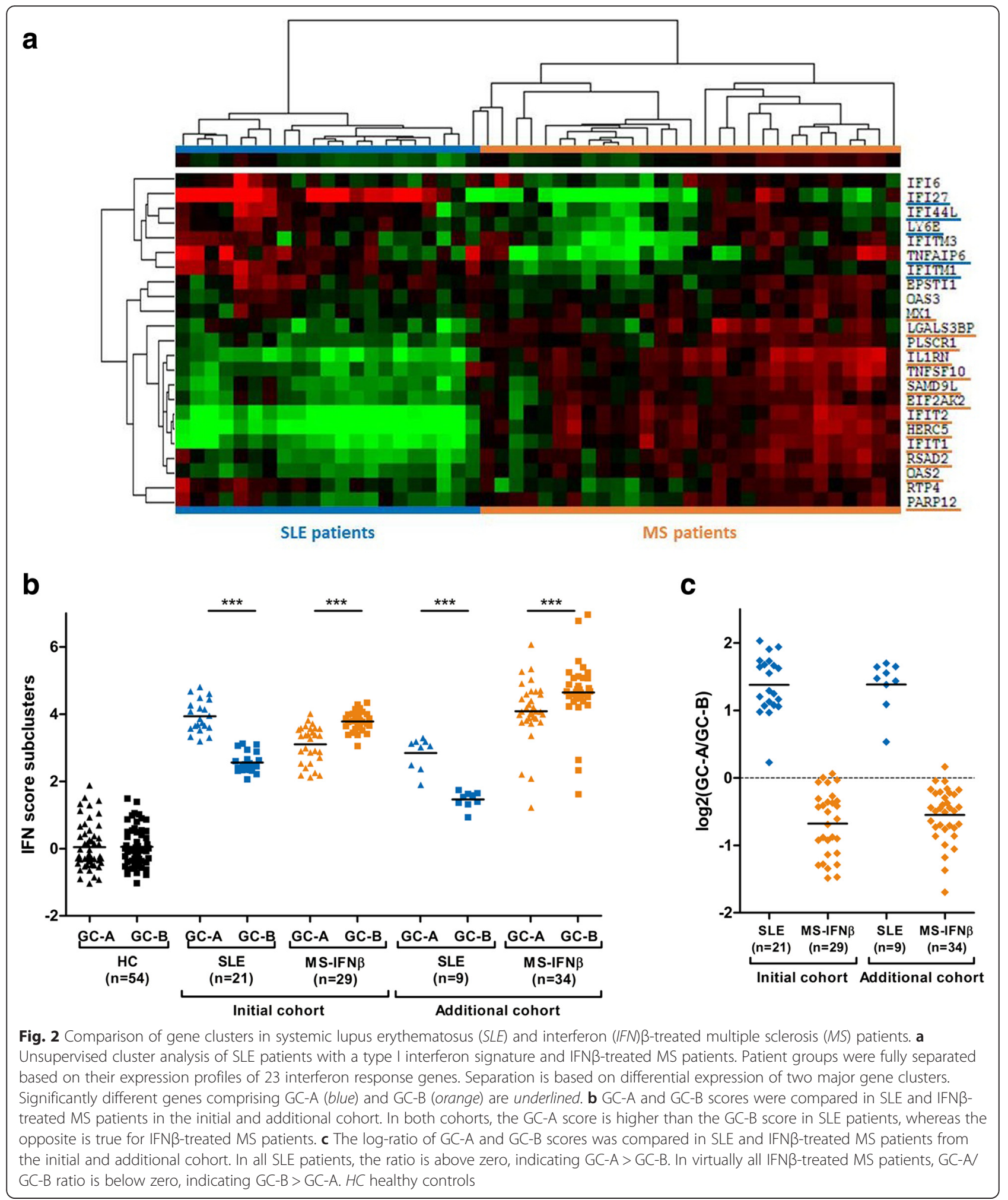

score is higher than the GC-A score. Comparison of these GC-A/GC-B log-ratios revealed that SLE and patients with MS from both cohorts could be completely separated based on these ratios (Fig. 2c).

\section{Validation in public microarray datasets}

In order to validate our observations, publicly available microarray data were downloaded from the Gene Expression Omnibus database of the National Center for 
Biotechnology Information [32]. Dataset GSE17755 contains gene expression data from peripheral blood cells from 25 healthy individuals and 22 SLE patients [33]. Datasets GSE41846 and GSE41848 consist of combined gene expression data from peripheral blood cells from 38 healthy controls and 78 IFN $\beta$-treated MS patients [34]. Expression data for the 23 IRGs were extracted from these datasets, except for $S A M D 9 L$ as it was not available in all sets. Patients with an IFN signature were selected based on the $\mathrm{HC}$ cutoff, as described above, and GC-A/GC-B log-ratios were determined. As shown in Fig. 3, these data confirmed our findings: SLE patients displayed a dominant GC-A score, whereas GC-B dominance was apparent for the majority (78 \%) of IFN $\beta$ treated MS patients. A small proportion of IFN $\beta$-treated MS patients had some GC-A dominance, which might be explained by the fact that we could not exclude patients with an IFN signature before start of therapy, as the dataset did not contain paired data before and during IFN $\beta$ treatment for all patients. Altogether, these data confirm the presence of IFN $\alpha$ - and IFN $\beta$-specific signatures and the utility of the GC-A/GC-B log-ratio to distinguish between those signatures.

Expression of gene clusters in other autoimmune diseases The above results indicate that IFN $\alpha$ and IFN $\beta$-driven type I IFN signatures can be distinguished based on the GC-A/GC-B log-ratio. Thereto, we determined the GC$\mathrm{A} / \mathrm{GC}-\mathrm{B} \log$-ratio in patients with idiopathic inflammatory myopathies (IIM), RA patients and IFN $\beta$-naive MS patients, which are autoimmune diseases with type I IFN signatures of yet unknown origin. Again, only IFN ${ }^{\text {high }}$ patients were selected.

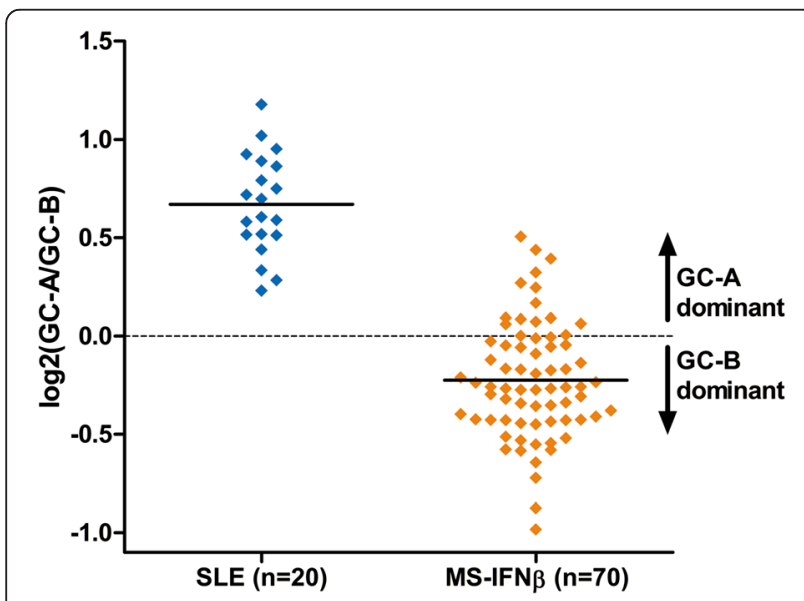

Fig. 3 Validation of our findings in publicly available microarray data. The ratio between GC-A and GC-B was calculated for independent validation cohorts of 20 systemic lupus erythematosus (SLE) patients with a type I interferon signature (IFN ${ }^{\text {high }}$ ) and 70 IFN ${ }^{\text {high }}$ IFN $\beta$-treated multiple sclerosis (MS) patients. This confirms our earlier findings of GC-A > GC-B in SLE and GC-B > GC-A in IFN $\beta$-treated MS
As shown in Fig. 4a, in patients with IIM there was GC-A dominance, as reflected by the positive GC-A/ GC-B log-ratios, indicating predominant IFN $\alpha$ activity similar to that in SLE. The GC-A/GC-B log-ratio in RA patients was lower and approached zero in some of the patients, indicating the contribution of the GC-B cluster as well. Remarkably, untreated MS patients appeared most heterogeneous; approximately half of the patients were characterized by GC-A dominance, whereas the other patients displayed GC-B dominance or a log-ratio close to zero.

These findings were validated for RA patients and untreated patients with MS who were IFN ${ }^{\text {high }}$, as the microarray datasets used for validation of our findings in SLE and IFN $\beta$-treated MS patients also contained gene expression data for RA patients (GSE17755, $\mathrm{n}=112$ ) and untreated patients with MS (GSE41846 and GSE41848, $\mathrm{n}=$ 62) (Fig. 4b) [33, 34].

\section{Transcriptional regulation of IRG gene clusters}

To explore functional differences between GC-A and GC-B genes, a transcription factor binding site (TFBS) analysis was performed on these gene clusters using the interferome database and rVISTA [35, 36]. Interestingly, output from the Interferome database showed that prototypical IFN-response elements IFNstimulated response element (ISRE), interferon consensus sequence-binding protein (ICSBP)/interferon regulatory factor (IRF) 8 and IRF7 are mainly present in the GC-B genes and not in the genes of GC-A (Fig. 5). This was supported by statistical analysis of TFBS enrichment, using rVista, which showed significant enrichment in GC-B of both IRF8-binding sites and ISRE (within a 100 bp upstream regulatory region, $p<0.0001$ and $p=0.02$, respectively). No enrichment of IFN-related TFBS was found in the GC-A gene set (data not shown). This indicates differential transcriptional regulation of the GC-A and GC-B genes, further supporting different upstream activity.

\section{Discussion}

Presence of a type I IFN signature is often discussed as a similarity among autoimmune diseases. In the present study we provide evidence that type I IFN signatures in autoimmune diseases appear less uniform than generally assumed.

IRG expression patterns were different between SLE and IFN $\beta$-treated MS, two autoimmune diseases in which IFN activity has opposing effects on immune pathology and regulation, believed to be a consequence of differential effects of IFN $\alpha$ and IFN $\beta$. Log-ratios of the differentially expressed type I IFN gene clusters, designated GC-A (SLE-IFN $\alpha$-related) and GC-B (MS-IFN $\beta$ related), revealed excellent separation of patients with 


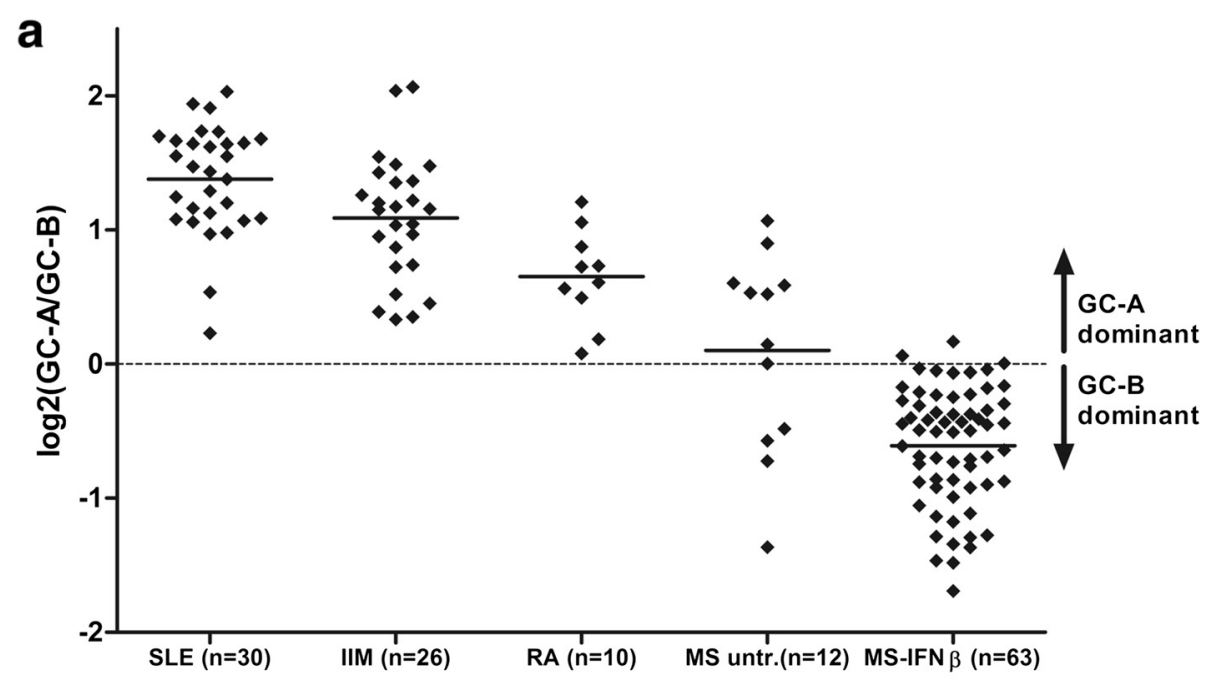

b

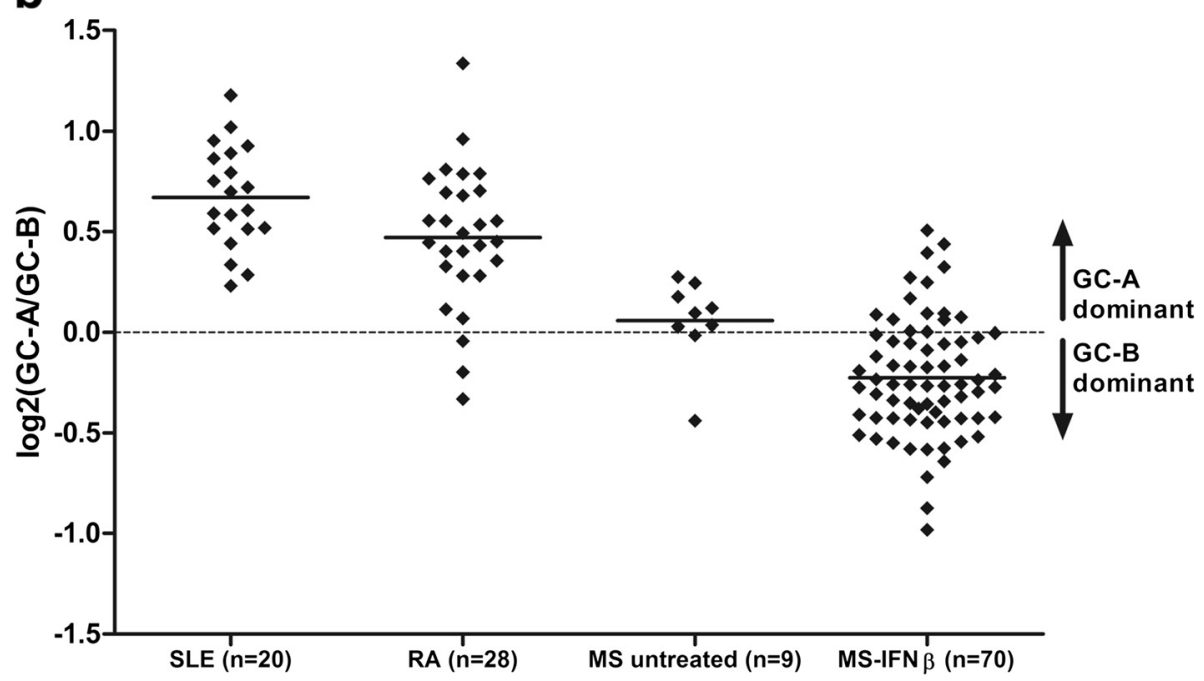

Fig. 4 Comparison of gene clusters in autoimmune diseases. a Log $_{2}(G C-A / G C-B)$ was compared between patients with systemic lupus erythematosus (SLE), untreated multiple sclerosis (MS) patients and patients with idiopathic inflammatory myopathies (IIM) or rheumatoid arthritis (RA). The GC-A/GC-B log-ratios are comparable in SLE and IIM. RA patients display less distinctive log-ratios for GC-A and GC-B. Untreated patients with MS are characterized by either GC-A or GC-B dominance. $\mathbf{b}$ Confirmation of these findings using publicly available microarray data. IFN interferon

SLE and IFN $\beta$-treated MS patients. Moreover, use of the GC-A/GC-B log-ratios in RA, IIM and IFN $\beta$-naïve MS patients provided insight into the origin of the type I IFN signatures in these diseases.

The GC-A/GC-B log-ratios revealed that IIM is predominantly characterized by a GC-A, hence an SLE-like, IFN signature. This fits with the many similarities between SLE and IIM that have been reported previously, including those related to the IFN pathway [37]. With regard to RA, the GC-A/GC-B log-ratio was close to zero for some patients, indicating that both IFN $\alpha$ and IFN $\beta$ contribute to the IFN signature, as has been suggested before [38]. Interestingly, GC-A/GC-B log-ratios differed among IFN $\beta$-naïve MS patients, as half of the patients had GC-A dominance, and others had GC-B dominance or ratios close to zero. This could implicate different mechanisms underlying the type I IFN pathway activation in these patients. We previously showed that presence of a baseline IFN signature in patients with MS is related to non-responsiveness to IFN $\beta$ treatment [27] and it is highly relevant to further investigate the role of GC-A and GC-B in this perspective, which is the objective of future studies. Altogether, these results suggest a differential role of type I IFNs in autoimmune diseases.

The considerable variance of type I IFNs in humans suggests that, although they bind to the same receptor, 


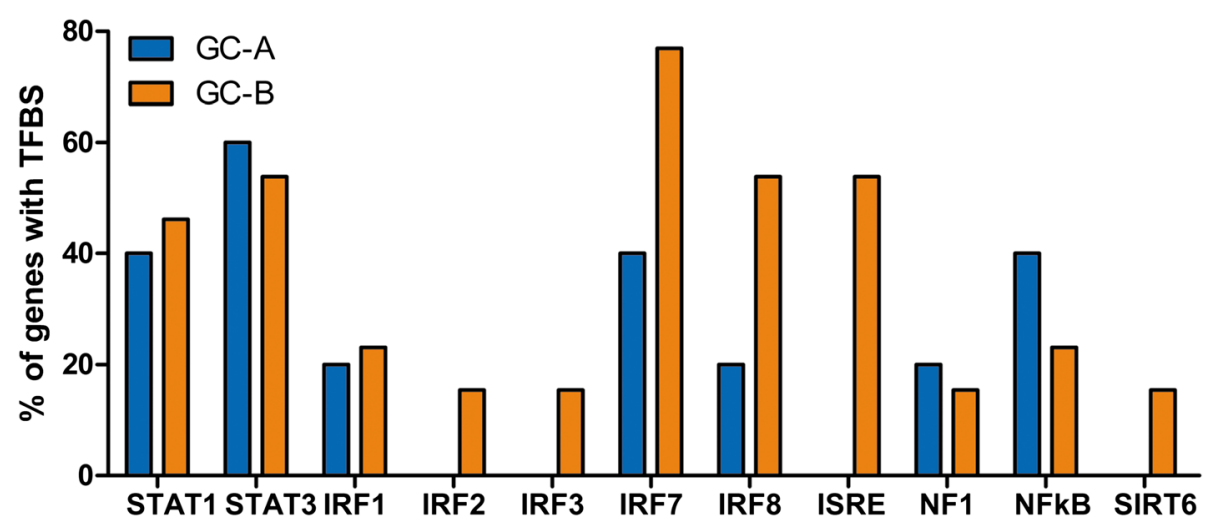

Fig. $\mathbf{5}$ Transcription factor binding site (TFBS) analysis using the Interferome database. Represents the presence of transcription factor binding elements $1500 \mathrm{bp}$ upstream from the transcription start site. Interferon regulatory factor (IRF)7, IRF8 and interferon-stimulated response element (ISRE) are mainly present in genes from GC-B and not in genes from GC-A

the effects they exert might be different. For example, it has been shown that IFN $\beta$ is more potent than IFN $\alpha$ in inhibiting proliferation, inducing apoptosis and cell differentiation [39, 40]. Differences were partly explained by the different affinities of type I IFNs for their receptors, resulting in different receptor trafficking, phosphorylation and signaling kinetics [21, 41]. More recently, it has been described that IFN $\beta$ can uniquely ligate to IFNAR1, independently of IFNAR2 [42]. Overall, IFN $\beta$ appears more potent in activating signal transduction than IFN $\alpha$, as demonstrated by a more stable receptor complex formation [43], a lower concentration of drug giving the half-maximal response $\left(\mathrm{EC}_{50}\right)$ for ISGF3 phosphorylation [44] and induction of a larger amount of genes than IFN $\alpha$, especially at long incubation times of 16-36 h [45]. Notably, these long incubation times conform to the chronic IFN $\alpha$ exposure in SLE and 3 months of IFN $\beta$ therapy in MS. Interestingly, Moraga et al. hypothesized that the short-term complex formation of IFN $\alpha$ with its receptors might cause a constant low level of apoptosis, whereas the long-term complex formation of IFN $\beta$ with its receptors could more potently induce high levels of apoptosis. As SLE pathology is characterized by impaired clearance of apoptotic cells, resulting in immune complex formation and consequent IFN $\alpha$ induction, the low levels of apoptosis as mediated by IFN $\alpha$ could be key to persistence of a vicious proinflammatory circle [46]. In MS, however, apoptosis of autoreactive $\mathrm{T}$ cells is considered to be one of the antiinflammatory actions of IFN $\beta$ therapy [47].

The implication of IFN $\alpha$-and IFN $\beta$-specific signatures is supported by the experiments of Der et al., who performed an in vitro experiment in which the fibrosarcoma cell line HT1080 was stimulated with either IFNa or IFN $\beta$, followed by gene expression measurements using oligonucleotide arrays for $\pm 6,800$ genes [48]. From these experiments, seven genes overlap with our gene clusters, one from the GC-A cluster and six from the GC-B cluster. IFITM1, a GC-A gene, had slightly higher expression in IFN $\alpha$-stimulated cells compared to IFN $\beta$, whereas the GC-B genes EIF2AK2, IFIT1, IFIT2, MX1,OAS2 and PLSCR1 were all induced more by IFN $\beta$ than by IFN $\alpha$ (1.2-fold to 7-fold higher induction) [48]. Despite the small overlap of genes, the consistency of these results is striking.

It has been suggested that the type I IFN response differs among immune cell types [49], implying that the observed differences between SLE and MS could be partly due to differences in immune cell composition. However, the agreement between our data and those of Der et al. suggests that the observed differences are due to consistent differential signaling in all cells rather than large differences in immune cell compositions or IFNAR expression. However, for replication and complete definition of IFN subtype-specific response programs, whole genome expression studies are required.

Analysis of transcriptional regulation of GC-A or GC$B$ genes showed enrichment of IRF8/ICSBP binding sites and ISRE in the GC-B cluster. Remarkably, none of the IRGs from the GC-A cluster contained an ISRE, the response element that binds the ISGF3 complex downstream of canonical type I IFN signaling. As they did contain binding sites for STAT1 and/or STAT2, they are probably induced via STAT1-STAT1 monomers or STAT1-STAT2 heterodimers, which both IFNa and IFN $\beta$ are able to activate $[50,51]$. The observation that these genes are increased in SLE compared to IFN $\beta$ treated MS might be explained by the indication that IFN $\beta$, in contrast to IFN $\alpha$, might more potently activate a broader range of signaling proteins, including ISGF3 and IRF8, resulting in relatively less activation of the GC-A genes by IFN $\beta$.

Expression of IRF8 is restricted to immune cells and it has the ability to act as a repressor or activator of the 
IFN response, depending on its interaction partner. A study by Meraro et al. reported that the IRF1-mediated induction of the IFN response gene ISG15 was inhibited in the presence of IRF8, whereas interaction of IRF8 and PU.1 synergistically enhanced ISG15 induction [52]. This suggests an immunomodulatory role for IRF8, which might be key to the different effects of IFN $\alpha$ and IFN $\beta$ on the immune system.

\section{Conclusions}

Conclusively, this study demonstrated that the IFN signatures display distinct differences between autoimmune diseases. Considering the pro-inflammatory nature of IFN $\alpha$ in SLE and the anti-inflammatory role of IFN $\beta$ in MS, specification of the type I IFN response in autoimmune diseases might give new insights into its role in disease pathology and/or its therapeutic potential.

\section{Additional file}

Additional file 1: Table S1. List of 23 type I interferon (IFN) response genes that were measured. Table S2. List of genes that are significantly differentially expressed between patients with systemic lupus erythematosus (SLE) and patients with multiple sclerosis (MS) who were treated with IFN $\beta$. (PDF $116 \mathrm{~kb}$ )

\section{Abbreviations}

bp: base pair(s); GAS: interferon-gamma activated sequence; HC: healthy control; ICSBP: interferon consensus sequence-binding protein; IFN: interferon; IFN ${ }^{\text {high }}$ : presence of a type I interferon signature; IIM: idiopathic inflammatory myopathies; IL: interleukin; IL-1R: interleuking-1 receptor; IRF: interferon regulatory factor; IRG: interferon response gene; ISGF: interferon-stimulated gene factor; ISRE: interferon-stimulated response element; MS: multiple sclerosis; RA: rheumatoid arthritis; SLE: systemic lupus erythematosus; TFBS: transcription factor binding site; TNF: tumor necrosis factor.

\section{Competing interests}

Dr. Hegen has participated in meetings sponsored by, or received honoraria (for lectures) from Bayer Schering, Biogen, Merck Serono and Novartis. Dr. Deisenhammer has participated in meetings sponsored by or received honoraria for acting as an advisor/speaker for Bayer Healthcare, Biogen Idec, Genzyme-Sanofi, Merck, Novartis Pharma, and Teva-Ratiopharm. His institution has received financial support for participation in randomized controlled trials of IFNb-1b (Betaferon, Bayer Schering Pharma), IFNb-1a (Avonex, Biogen Idec; Rebif, Merck Serono), glatiramer acetate (Copaxone, Teva Pharmaceuticals), Natalizumab (Tysabri, Biogen Idec), in multiple sclerosis. He is section editor of the Multiple Sclerosis and Related Disorders (MSARD) journal. Dr. Killestein has accepted consulting fees from Merck-Serono, Biogen Idec, Teva, Genzyme and Novartis. The other authors have no competing interests to declare.

\section{Authors' contributions}

All authors were involved in drafting the article or revising it critically for important intellectual content, and all authors approved the final version to be published. CV had full access to all of the data in the study and takes responsibility for the integrity of the data and the accuracy of the data analysis. Study concept and design: TdJ, SV and CV. Acquisition of patient material and of data: TdJ, SV, EM, JW, CL, HH, FD, JK, IL, JV, MN, DVS, IB and AV. Analysis and interpretation of data: TdJ, SV, SdR, TPK, MP, CVdL, JB and CV.

\section{Acknowledgements}

This research was performed with support from the Dutch Arthritis Foundation (project number 13-2-307) and the Dutch MS Research Foundation (grant 04-
549 MS). The NABINMS study was supported by the European Union (grant number LSHB-CT-2005-018926). The Sweden IIM cohort was supported by The Swedish Research Council, the Swedish Rheumatism Association, the European Union Sixth Framework Program (project AutoCure; LSH-018661) and the European Science Foundation. The Czech IIM cohort was supported by Project for Conceptual Development of Research Organization 00023728 from the Ministry of Health in the Czech Republic.

\section{Author details}

${ }^{1}$ Department of Pathology, VU University Medical Center, Amsterdam, The Netherlands. ${ }^{2}$ Department of Molecular Cell Biology and Immunology, VU University Medical Center, Amsterdam, The Netherlands. ${ }^{3}$ Department of Neurology, VU University Medical Center, Amsterdam, The Netherlands. ${ }^{4}$ Department of Neurology, Innsbruck Medical University, Innsbruck, Austria. ${ }^{5}$ Rheumatology Unit, Department of Medicine, Karolinska University Hospital, Solna, Karolinska Institutet, Stockholm, Sweden. ${ }^{6}$ Institute of Rheumatology, Prague, Czech Republic. ${ }^{7}$ Department of Rheumatology, Amsterdam Rheumatology and Immunology Center, Reade, Amsterdam, The Netherlands. ${ }^{8}$ Department of Rheumatology, Amsterdam Rheumatology and Immunology Center, VUmc, Amsterdam, The Netherlands.

Received: 12 October 2015 Accepted: 1 February 2016 Published online: 17 February 2016

\section{References}

1. Stark GR, Kerr IM, Williams BR, Silverman RH, Schreiber RD. How cells respond to interferons. Annu Rev Biochem. 1998;67:227-64.

2. Takaoka A, Taniguchi T. New aspects of IFN-alpha/beta signalling in immunity, oncogenesis and bone metabolism. Cancer Sci. 2003;94:405-11.

3. Ghislain JJ, Fish EN. Application of genomic DNA affinity chromatography identifies multiple interferon-alpha-regulated Stat2 complexes. J Biol Chem. 1996;271:12408-13.

4. Li X, Leung S, Qureshi S, Darnell Jr JE, Stark GR. Formation of STAT1-STAT2 heterodimers and their role in the activation of IRF-1 gene transcription by interferon-alpha. J Biol Chem. 1996;271:5790-4.

5. Borden EC, Sen GC, Uze G, Silverman RH, Ransohoff RM, Foster GR, et al. Interferons at age 50: past, current and future impact on biomedicine. Nat Rev Drug Discov. 2007;6:975-90.

6. Randall RE, Goodbourn S. Interferons and viruses: an interplay between induction, signalling, antiviral responses and virus countermeasures. J Gen Virol. 2008:89:1-47.

7. Higgs BW, Liu Z, White B, Zhu W, White WI, Morehouse C, et al. Patients with systemic lupus erythematosus, myositis, rheumatoid arthritis and scleroderma share activation of a common type I interferon pathway. Ann Rheum Dis. 2011;70:2029-36.

8. Bave U, Nordmark G, Lovgren T, Ronnelid J, Cajander S, Eloranta ML, et al. Activation of the type I interferon system in primary Sjogren's syndrome: a possible etiopathogenic mechanism. Arthritis Rheum. 2005; 52:1185-95

9. van Baarsen LG, van der Pouw Kraan TC, Kragt JJ, Baggen JM, Rustenburg F, Hooper T, et al. A subtype of multiple sclerosis defined by an activated immune defense program. Genes Immun. 2006;7:522-31.

10. Blanco P, Palucka AK, Gill M, Pascual V, Banchereau J. Induction of dendritic cell differentiation by IFN-alpha in systemic lupus erythematosus. Science. 2001;294:1540-3.

11. Kirou KA, Lee C, George S, Louca K, Peterson MG, Crow MK. Activation of the interferon-alpha pathway identifies a subgroup of systemic lupus erythematosus patients with distinct serologic features and active disease. Arthritis Rheum. 2005;52:1491-503.

12. Ronnblom L, Alm GV, Eloranta ML. The type I interferon system in the development of lupus. Semin Immunol. 2011;23:113-21.

13. Bennett L, Palucka AK, Arce E, Cantrell V, Borvak J, Banchereau J, et al. Interferon and granulopoiesis signatures in systemic lupus erythematosus blood. J Exp Med. 2003;197:711-23.

14. Dall'era MC, Cardarelli PM, Preston BT, Witte A, Davis Jr JC. Type I interferon correlates with serological and clinical manifestations of SLE. Ann Rheum Dis. 2005:64:1692-7.

15. Ronnblom LE, Alm GV, Oberg KE. Possible induction of systemic lupus erythematosus by interferon-alpha treatment in a patient with a malignant carcinoid tumour. J Intern Med. 1990;227:207-10. 
16. Ho V, Mclean A, Terry S. Severe systemic lupus erythematosus induced by antiviral treatment for hepatitis C. J Clin Rheumatol. 2008;14:166-8.

17. Hafler DA, Slavik JM, Anderson DE, O'Connor KC, de Jager P, Baecher-Allan C. Multiple sclerosis. Immunol Rev. 2005;204:208-31.

18. Interferon beta-1b in the treatment of multiple sclerosis: final outcome of the randomized controlled trial. The IFNB Multiple Sclerosis Study Group and The University of British Columbia MS/MRI Analysis Group. Neurology. 1995; 45:1277-85.

19. Chawla-Sarkar M, Lindner DJ, Liu YF, Williams BR, Sen GC, Silverman RH, et al. Apoptosis and interferons: role of interferon-stimulated genes as mediators of apoptosis. Apoptosis. 2003;8:237-49.

20. Taniguchi T, Mantei N, Schwarzstein M, Nagata S, Muramatsu M, Weissmann C. Human leukocyte and fibroblast interferons are structurally related. Nature. 1980;285:547-9.

21. Jaitin DA, Roisman LC, Jaks E, Gavutis M, Piehler J, Van der Heyden J, et al. Inquiring into the differential action of interferons (IFNs): an IFN-alpha2 mutant with enhanced affinity to IFNAR1 is functionally similar to IFN-beta. Mol Cell Biol. 2006;26:1888-97.

22. Lewerenz M, Mogensen KE, Uze G. Shared receptor components but distinct complexes for alpha and beta interferons. J Mol Biol. 1998; 282:585-99.

23. Platanias LC, Uddin S, Domanski P, Colamonici OR. Differences in interferon alpha and beta signaling. Interferon beta selectively induces the interaction of the alpha and betaL subunits of the type I interferon receptor. J Biol Chem. 1996;271:23630-3.

24. Johns TG, Mackay IR, Callister KA, Hertzog PJ, Devenish RJ, Linnane AW. Antiproliferative potencies of interferons on melanoma cell lines and xenografts: higher efficacy of interferon beta. J Natl Cancer Inst. 1992;84: 1185-90.

25. Hegen H, Millonig A, Bertolotto A, Comabella M, Giovanonni G, Guger M, et al. Early detection of neutralizing antibodies to interferon-beta in multiple sclerosis patients: binding antibodies predict neutralizing antibody development. Mult Scler. 2014;20:577-87.

26. Baechler EC, Batliwalla FM, Karypis G, Gaffney PM, Ortmann WA, Espe KJ, et al. Interferon-inducible gene expression signature in peripheral blood cells of patients with severe lupus. Proc Natl Acad Sci USA. 2003;100:2610-5.

27. van Baarsen LG, Vosslamber S, Tijssen M, Baggen JM, van der Voort LF, Killestein J, et al. Pharmacogenomics of interferon-beta therapy in multiple sclerosis: baseline IFN signature determines pharmacological differences between patients. PLoS One. 2008:3:e1927.

28. van der Pouw Kraan TC, Wijbrandts CA, van Baarsen LG, Voskuyl AE, Rustenburg F, Baggen JM, et al. Rheumatoid arthritis subtypes identified by genomic profiling of peripheral blood cells: assignment of a type I interferon signature in a subpopulation of patients. Ann Rheum Dis. 2007; 66:1008-14.

29. Raterman HG, Vosslamber S, de Ridder S, Nurmohamed MT, Lems WF, Boers $M$, et al. The interferon type I signature towards prediction of non-response to rituximab in rheumatoid arthritis patients. Arthritis Res Ther. 2012;14:R95.

30. Greenberg SA, Higgs BW, Morehouse C, Walsh RJ, Kong SW, Brohawn P, et al. Relationship between disease activity and type 1 interferon- and other cytokine-inducible gene expression in blood in dermatomyositis and polymyositis. Genes Immun. 2012;13:207-13.

31. Eisen MB, Spellman PT, Brown PO, Botstein D. Cluster analysis and display of genome-wide expression patterns. Proc Natl Acad Sci USA. 1998;95:14863-8.

32. Edgar R, Domrachev M, Lash AE. Gene Expression Omnibus: NCBI gene expression and hybridization array data repository. Nucleic Acids Res. 2002; 30:207-10.

33. Lee HM, Sugino $H$, Aoki C, Nishimoto N. Underexpression of mitochondrialDNA encoded ATP synthesis-related genes and DNA repair genes in systemic lupus erythematosus. Arthritis Res Ther. 2011;13:R63.

34. Nickles D, Chen HP, Li MM, Khankhanian P, Madireddy L, Caillier SJ, et al. Blood RNA profiling in a large cohort of multiple sclerosis patients and healthy controls. Hum Mol Genet. 2013;22:4194-205.

35. Rusinova I, Forster S, Yu S, Kannan A, Masse M, Cumming H, et al. Interferome v2.0: an updated database of annotated interferon-regulated genes. Nucleic Acids Res. 2013;41:D1040-6.

36. Frazer KA, Pachter L, Poliakov A, Rubin EM, Dubchak I. VISTA: computational tools for comparative genomics. Nucleic Acids Res. 2004;32:W273-9.

37. Eloranta ML, Barbasso HS, Ulfgren AK, Ronnblom L, Alm GV, Lundberg IE. A possible mechanism for endogenous activation of the type I interferon system in myositis patients with anti-Jo-1 or anti-Ro 52/anti-Ro 60 autoantibodies. Arthritis Rheum. 2007:56:3112-24.

38. Mavragani CP, La DT, Stohl W, Crow MK. Association of the response to tumor necrosis factor antagonists with plasma type I interferon activity and interferon-beta/alpha ratios in rheumatoid arthritis patients: a post hoc analysis of a predominantly Hispanic cohort. Arthritis Rheum. 2010;62: 392-401.

39. Platanias LC, Uddin S, Colamonici OR. Tyrosine phosphorylation of the alpha and beta subunits of the type I interferon receptor. Interferon-beta selectively induces tyrosine phosphorylation of an alpha subunit-associated protein. J Biol Chem. 1994;269:17761-4.

40. Deonarain R, Verma A, Porter AC, Gewert DR, Platanias LC, Fish EN. Critical roles for IFN-beta in lymphoid development, myelopoiesis, and tumor development: links to tumor necrosis factor alpha. Proc Natl Acad Sci USA. 2003;100:13453-8

41. Marijanovic Z, Ragimbeau J, Van der Heyden J, Uze G, Pellegrini S. Comparable potency of IFNalpha2 and IFNbeta on immediate JAK/STAT activation but differential down-regulation of IFNAR2. Biochem J. 2007:407:141-51.

42. de Weerd NA, Vivian JP, Nguyen TK, Mangan NE, Gould JA, Braniff SJ, et al. Structural basis of a unique interferon-beta signaling axis mediated via the receptor IFNAR1. Nat Immunol. 2013;14:901-7.

43. Moraga I, Harari D, Schreiber G, Uze G, Pellegrini S. Receptor density is key to the alpha2/beta interferon differential activities. Mol Cell Biol. 2009;29: 4778-87.

44. Jaks E, Gavutis M, Uze G, Martal J, Piehler J. Differential receptor subunit affinities of type I interferons govern differential signal activation. J Mol Biol. 2007;366:525-39.

45. Piehler J, Thomas C, Garcia KC, Schreiber G. Structural and dynamic determinants of type I interferon receptor assembly and their functional interpretation. Immunol Rev. 2012;250:317-34.

46. Munoz LE, van Bavel C, Franz S, Berden J, Herrmann M, van der Vlag J. Apoptosis in the pathogenesis of systemic lupus erythematosus. Lupus. 2008;17:371-5.

47. Dhib-Jalbut S, Marks S. Interferon-beta mechanisms of action in multiple sclerosis. Neurology. 2010;74 Suppl 1:S17-24.

48. Der SD, Zhou A, Williams BR, Silverman RH. Identification of genes differentially regulated by interferon alpha, beta, or gamma using oligonucleotide arrays. Proc Natl Acad Sci USA. 1998;95:15623-8.

49. van Boxel-Dezaire AH, Zula JA, Xu Y, Ransohoff RM, Jacobberger JW, Stark GR. Major differences in the responses of primary human leukocyte subsets to IFN-beta. J Immunol. 2010;185:5888-99.

50. Ghislain JJ, Wong T, Nguyen M, Fish EN. The interferon-inducible Stat2:Stat1 heterodimer preferentially binds in vitro to a consensus element found in the promoters of a subset of interferon-stimulated genes. J Interferon Cytokine Res. 2001;21:379-88.

51. Brierley MM, Fish EN. Functional relevance of the conserved DNA-binding domain of STAT2. J Biol Chem. 2005;280:13029-36.

52. Meraro D, Gleit-Kielmanowicz M, Hauser H, Levi BZ. IFN-stimulated gene 15 is synergistically activated through interactions between the myelocyte/ lymphocyte-specific transcription factors, PU.1, IFN regulatory factor-8/IFN consensus sequence binding protein, and IFN regulatory factor-4: characterization of a new subtype of IFN-stimulated response element. J Immunol. 2002;168:6224-31. 